\title{
Recent Results from NA48/2 Experiment at CERN-SPS
}

\author{
Simone Bifani ${ }^{1}$ \\ University of Torino, Experimental Physics Department \\ via Pietro Giuria 1, I-10125 - Torino (Italy)
}

\begin{abstract}
Preliminary results of the measurement of charged CP violating asymmetry $A_{g}=\frac{g^{+}-g^{-}}{g^{+}+g^{-}}$ in both $3 \pi$ decay modes, based on the whole accumulated statistics in 2003 and 2004, are presented. A new unexpected anomaly in the $2 \pi^{0}$ mass subsystem spectrum in $K^{ \pm} \rightarrow \pi^{ \pm} \pi^{0} \pi^{0}$ decay, so called "cusp", due to charge exchange re-scattering process $\left(\pi^{+} \pi^{-} \rightarrow \pi^{0} \pi^{0}\right)$ is discussed.

A preliminary result indicates the observation of direct emission amplitude and first observation of an interference term in the non-leptonic radiative rare decay $K^{ \pm} \rightarrow \pi^{ \pm} \pi^{0} \gamma$ is presented.
\end{abstract}

Keywords: <charged kaon, direct cp violation, scattering length, radiative decay>

PACS: <13.25.Es >

\section{INTRODUCTION}

The NA48/2 experiment at CERN-SPS collected a very high statistics of charged kaon decays with the main purpose of searching for direct CP violation in $K^{ \pm} \rightarrow \pi^{ \pm} \pi^{+} \pi^{-}$ and $K^{ \pm} \rightarrow \pi^{ \pm} \pi^{0} \pi^{0}$ final states. Data were taken in 2003 and 2004 for a total amount of $4 \times 10^{9} K^{ \pm} \rightarrow \pi^{ \pm} \pi^{+} \pi^{-}$and $0.1 \times 10^{9} K^{ \pm} \rightarrow \pi^{ \pm} \pi^{0} \pi^{0}$. The total accumulated statistics allows to measure rare decays down to $\sim 10^{-9}$ branching ratios.

\section{NA48/2 EXPERIMENTAL SETUP}

NA48/2 is a fixed target experiment located at the CERN-SPS which adds a novel beam line system and a new beam spectrometer to the existing NA48 detector in order to reach a high accuracy in the measurement of the $\mathrm{CP}$ violating charge asymmetry parameter $A_{g}$ and rare kaon decays. The layout of the beams and detectors is shown schematically in Fig.1.

Two opposite in charge beams, $\mathrm{K}^{+}$and $\mathrm{K}^{-}$, are produced with protons at $400 \mathrm{GeV} / \mathrm{c}$ from SPS impinging on a beryllium target and then momentum selected in the band $(60 \pm 3) \mathrm{GeV} / \mathrm{c}$. Both beams are sent in the KAonBEamSpectrometer, a Micromega type detector operating in TPC mode which provides a $1 \%$ momentum measurement. The beams travel superimposed in space within $\sim 1 \mathrm{~mm}$ and then sent to the decay tank $(\sim 114 m)$. The $K^{+} / K^{-}$flux ratio is $\sim 1.8$.

\footnotetext{
1 on behalf of the NA48/2 Collaboration: Cambridge, CERN, Chicago, Dubna, Edinburgh, Ferrara,
} Firenze, Mainz, Northwestern, Perugia, Pisa, Saclay, Siegen, Torino, Vienna 


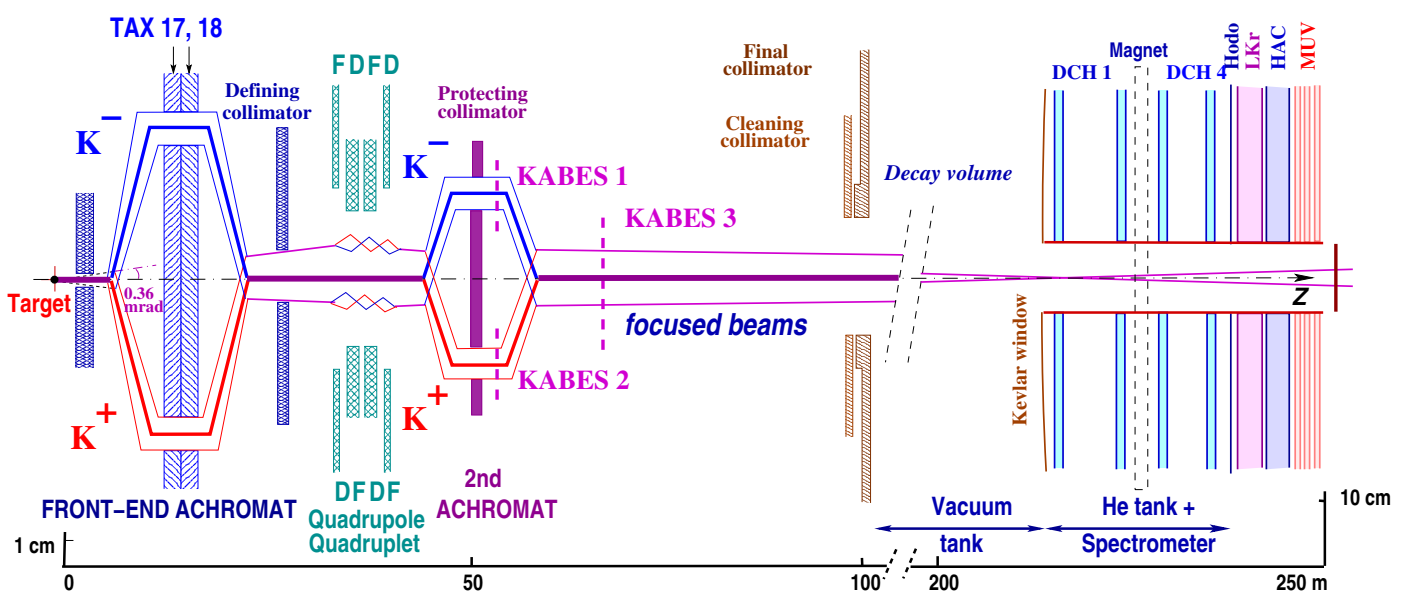

FIGURE 1. Schematic lateral view of the NA48/2 beam line, decay volume and detector (the vertical scale changes for the two parts of the figure).

Charged particles are measured using the magnetic spectrometer which is built with four drift chambers $(\mathrm{DCH})$ and one dipole magnet (horizontal momentum kick of 120 $\mathrm{MeV} / \mathrm{c}$ ) with a momentum resolution of $\sigma(p) / p=0.01 \oplus 0.00044 \cdot p(p$ in $\mathrm{GeV} / \mathrm{c})$. The magnetic spectrometer is followed by a scintillator hodoscope (HODO) consisting of two planes segmented into horizontal and vertical strips and arranged in four quadrants. Neutral particles are reconstructed by a liquid krypton electromagnetic calorimeter (LKr), a quasi-homogeneous ionization chamber $\left(\sim 27 X_{0}\right)$, which has an energy resolution of $\sigma(E) / E=0.032 / \sqrt{E} \oplus 0.09 / E \oplus 0.0042$ and a spatial resolution of $\sigma_{x}=\sigma_{y}=$ $0.42 / \sqrt{E} \oplus 0.06 \mathrm{~cm}$ ( $E$ in $\mathrm{GeV}$ ). At a depth of $\sim 9.5 X_{0}$ inside the active volume of the calorimeter, a hodoscope consisting of a plane of scintillating fibres is installed. A two level trigger is used to reduce the event rate from $\sim 500 \mathrm{kHz}$ to $\sim 10 \mathrm{kHz}$.

A detailed description of the detector can be found elsewhere [3].

\section{CP VIOLATING CHARGE ASYMMETRY}

More than thirty years have passed since the first discovery of indirect $\mathrm{CP}$ violation in the neutral kaon system [1] to also establish the existence of direct CP violation $[2,3,4]$. A measurement of that kind of violation in all systems where it is possible is quite relevant to test the Standard Model. In the kaon system the most promising complementary observables are decay rates of GIM suppressed rare kaon decays proceeding through flavour-changing neutral currents $\left(K^{ \pm} \rightarrow \pi^{ \pm} v \bar{v}\right)$ and the charge asymmetry between $K^{+}$and $K^{-}$decays in three pions.

The standard phenomenological description of $K^{ \pm} \rightarrow 3 \pi$ decays [5] is made in terms of the bi-dimensional Dalitz plot parameters $u$ and $v$, related respectively to the energy sharing to the "odd" pion (charge opposite with respect to the other two) and among the two "even" pions (same charge): 


$$
u=\frac{s_{3}-s_{0}}{m_{\pi}^{2}} \quad v=\frac{s_{2}-s_{1}}{m_{\pi}^{2}}
$$

where $s_{i}=\left(P_{K}-P_{\pi_{i}}\right)^{2}, i=1,2,3, s_{0}=\frac{s_{1}+s_{2}+s_{3}}{3}$ being $P_{K}$ and $P_{\pi_{i}}$ the kaon and pion four-momenta, the indexes $i=1,2$ correspond to the two identical pions and the index $i=3$ to the pion of different charge.

The matrix element is usually described in a polynomial expansion of the two Dalitz variables and parametrized in terms of slopes [5]:

$$
|M(u, v)|^{2} \approx 1+g u+h u^{2}+k v^{2}
$$

where $g\left(\pi^{ \pm} \pi^{+} \pi^{-}\right)=-0.2154 \pm 0.0035, g\left(\pi^{ \pm} \pi^{0} \pi^{0}\right)=-0.638 \pm 0.020$ and $|h|,|k| \ll$ $|g|$. A linear term in $v$ is forbidden due to symmetry considerations.

A difference of slope parameters $g^{+}$and $g^{-}$describing positive and negative kaon decays respectively, is a manifestation of direct $\mathrm{CP}$ violation usually defined by the corresponding slope asymmetry:

$$
A_{g}=\frac{g^{+}-g^{-}}{g^{+}+g^{-}} \approx \frac{\Delta g}{2 g}
$$

where $\Delta g$ is the slope difference and $g$ is the average slope. SM predictions [6] for that asymmetry vary between few $10^{-6}$ to few $10^{-5}$. Models beyond the SM [7] predict a substantial enhancement of $A_{g}$ that, while being out of reach for previous experiments [8] with lower statistics, are potentially accessible to NA48/2.

The measurement method is based on comparing the reconstructed $u$ distributions of $K^{+}$and $K^{-}$decays $\left(N^{+}(u)\right.$ and $\left.N^{-}(\mathrm{u})\right)$ : in the $K^{ \pm} \rightarrow \pi^{ \pm} \pi^{+} \pi^{-}$case the ratio $R(u)=$ $N^{+}(u) / N^{-}(u)$ is in good approximation proportional to $(1+\Delta g \cdot u)$, so $\Delta g$ can be extracted from a linear fit and $A_{g}=\Delta g / 2 g$ can be evaluated.

Charge symmetrization of the experimental conditions is to a large extent achieved by using simultaneous and collinear $K^{+}$and $K^{-}$beams with similar momentum spectra and reversing all the magnets polarities during the data taking: beam line magnets on weekly basis while spectrometer magnet once per day in 2003 and once in 3 hours in 2004. Data collected over a period with all the four possible magnets setup configurations represent a "SuperSample", which is treated as an independent and self-consistent set of data for asymmetry measurement (nine SuperSamples were collected in two years of data taking). To measure the charge asymmetry the following "quadruple ratio" composed as a product of four $R(u)=N^{+}(u) / N^{-}(u)$ ratios with opposite kaon sign, and deliberately chosen setup configurations in numerator and denominator, is considered:

$$
R_{4}(u)=R_{U S}(u) \cdot R_{U J}(u) \cdot R_{D S}(u) \cdot R_{D J}(u)
$$

where the indices $U(D)$ denote the beam line polarities corresponding to $K^{+}$passing along the upper (lower) path in the achromats, respectively, while the indices $S(J)$ represent spectrometer magnet polarities (opposite for $\mathrm{K}^{+}$and $\mathrm{K}^{-}$) corresponding to the "even" pions being deflected to negative (positive) horizontal coordinate, i.e. towards the Salève (Jura) mountains, respectively. 
The quadruple ratio technique logically completes the procedure of magnet polarity reversal, and allows a three-fold cancelation of systematic biases:

- beam line differences: by comparing $K^{+}$and $K^{-}$traveling along the same paths;

- detector asymmetries: by comparing $K^{+}$and $K^{-}$illuminating the detector in the same way;

- global time-dependent effects: by the simultaneous detection of $K^{+}$and $K^{-}$events.

The result remains sensitive only to time variations of asymmetries in the experimental conditions which have a characteristic time smaller than corresponding field alternation period, and in principle should be free of systematic biases.

The method is independent of the $K^{+} / K^{-}$flux ratio and the relative sizes of the samples collected with different magnet configurations, however the statistical precision is limited by the smallest of the samples involved, so the balance of sample sizes was controlled during the data taking.

Due to the method described above, no Monte-Carlo corrections to the acceptance are expected to be needed, nevertheless a detailed GEANT-based Monte-Carlo simulation was developed as a tool for systematic studies, including full detector geometry and material description, simulation of time-variable local DCH inefficiencies, time variations of the beam geometry and DCH alignment.

\section{"Charged" Mode: $K^{ \pm} \rightarrow \pi^{ \pm} \pi^{+} \pi^{-}$}

Tracks are reconstructed from hits in DCHs using the measured magnetic field map of the spectrometer magnet rescaled according to the recorded current. Three-track vertices compatible with a $K^{ \pm} \rightarrow \pi^{ \pm} \pi^{+} \pi^{-}$decay topology are reconstructed by extrapolation of track segments from the spectrometer upstream to the decay volume, taking into account the small magnetic field due to residual vacuum tank magnetization and the Earth's field, which were measured before the run. Event selection includes requirements on vertex charge, quality, position, limits on the reconstructed $3 \pi$ momentum $\left(54 \mathrm{GeV} / \mathrm{c}<p_{K}<\right.$ $66 \mathrm{GeV} / \mathrm{c}$ ) and invariant mass $\left(\left|m_{\pi \pi \pi}-m_{K}<9 \mathrm{MeV} / \mathrm{c}^{2}\right|\right)$. The reconstructed $3 \pi$ mass distribution tails are dominated by events in which a pion undergoes a $\pi \rightarrow \mu \nu$ decay but no muon rejection is applied to avoid introducing instrumental asymmetries. The selection leaves a practically background free sample $\left(3.11 \times 10^{9} \mathrm{~K}^{ \pm}\right.$decays selected from the total 2003 and 2004 data sample), as $K^{ \pm} \rightarrow \pi^{ \pm} \pi^{+} \pi^{-}$is the dominant threetrack $K^{ \pm}$decay mode.

Possible sources of systematic effects were studied and evaluated such as the fine alignment of the spectrometer, the geometrical acceptance seen by the two beams, the dependence on the way the $u$ variable is calculated or the fitting limits, effects due to uncertainty on the knowledge of the magnetic fields, pile-up effects, inhomogeneities in the spectrometer alignment and trigger efficiencies (Tab.1).

After applying the above corrections, $\Delta g$ is extracted by fitting the quadruple ratio of the $u$ spectra for each SuperSample: the independent obtained results are compatible with $\chi^{2} / n d f=10.0 / 8$ (Fig.2). 
TABLE 1. Systematic uncertainties.

\begin{tabular}{l|c}
\hline Systematic effect & Correction, uncertainty $\delta(\Delta g) \times 10^{4}$ \\
\hline Spectrometer alignment & \pm 0.1 \\
Acceptance and beam geometry & \pm 0.2 \\
Momentum scale & \pm 0.1 \\
Pion decay & \pm 0.4 \\
Pile-up & \pm 0.2 \\
Resolution and fitting & \pm 0.3 \\
\hline Total systematic uncertainty & \pm 0.6 \\
\hline Level 1 trigger & \pm 0.3 \\
Level 2 trigger & $0.1 \pm 0.3$ \\
\hline
\end{tabular}

The difference in $K^{ \pm} \rightarrow \pi^{ \pm} \pi^{+} \pi^{-}$Dalitz plot slope parameter is found to be:

$$
\Delta g=\left(0.6 \pm 0.7_{\text {stat }} \pm 0.4_{\text {trig }} \pm 0.6_{\text {syst }}\right) \times 10^{-4}=(0.6 \pm 1.0) \times 10-4
$$

leading to a $\mathrm{CP}$ violating charge asymmetry using $g=-0.2154 \pm 0.0035$ [5]:

$$
A_{g}=\left(-1.3 \pm 1.5_{\text {stat }} \pm 0.9_{\text {trig }} \pm 1.4_{\text {syst }}\right) \times 10^{-4}=(-1.3 \pm 2.3) \times 10^{-4}
$$

which does not contradict the SM, and due to high precision can be used to constrain SM extensions predicting enhancements of the charge asymmetry. The result has $\sim 17$ times better precision than the best measurement before NA48/2, and the precision is still limited mainly by the available statistics (the uncertainty due to the trigger inefficiency is of statistical nature).

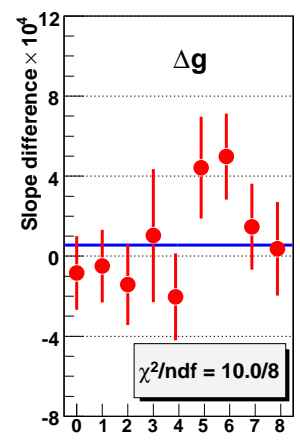

FIGURE 2. $\Delta g$ measurement in the nine SuperSamples.

$$
\text { "Neutral" Mode: } K^{ \pm} \rightarrow \pi^{ \pm} \pi^{0} \pi^{0}
$$

An analogous analysis in the "neutral" mode using $91 \times 10^{6} K^{ \pm}$decays, from the combined 2003 and 2004 data sample, gives the result:

$$
A_{g}=\left(2.1 \pm 1.6_{\text {stat }} \pm 1.0_{\text {syst }} \pm 0.2_{\text {ext }}\right) \times 10^{-4}=(2.1 \pm 1.9) \times 10^{-4}
$$

The statistical precision is similar with respect to the "charged" mode because, even though the $K^{ \pm} \rightarrow \pi^{ \pm} \pi^{+} \pi^{-}$decay is statistically favored due to higher branching ratio and acceptance, the population density of the Dalitz plot is less favorable and $\left|g\left(\pi^{ \pm} \pi^{0} \pi^{0}\right)\right| \sim 3 \cdot\left|g\left(\pi^{ \pm} \pi^{+} \pi^{-}\right)\right|$. 

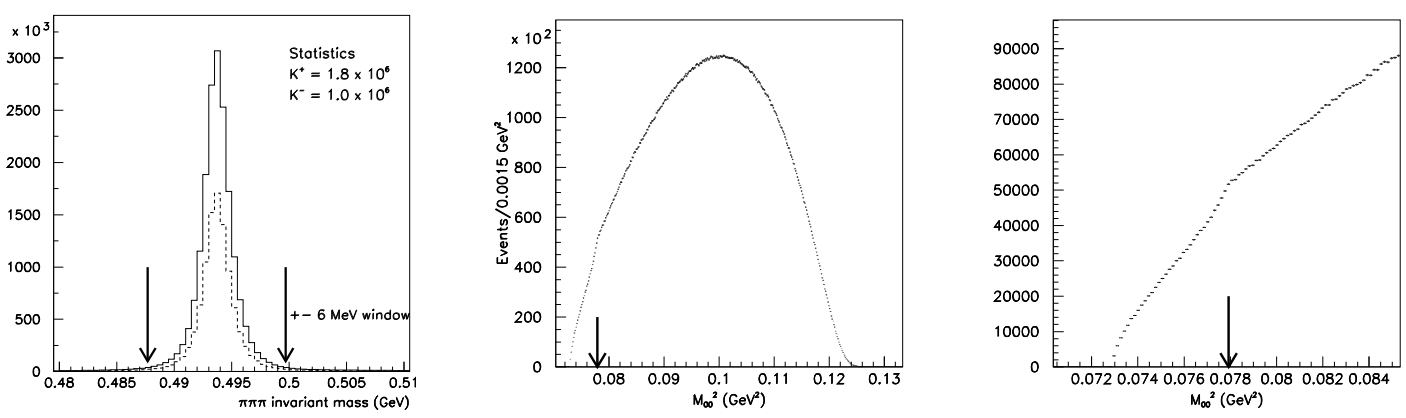

FIGURE 3. (Left) Invariant mass distribution of $K^{ \pm} \rightarrow \pi^{ \pm} \pi^{0} \pi^{0}$ candidate events. (Middle) $\pi^{0} \pi^{0}$ invariant mass squared distribution. (Right) Zoom of the previous in the region around the value $M_{00}^{2}=$ $\left(2 m_{+}\right)^{2}=0.07792\left(\mathrm{GeV} / \mathrm{c}^{2}\right)^{2}$ indicated by the arrow.

\section{"CUSP" EFFECT IN $K^{ \pm} \rightarrow \pi^{ \pm} \pi^{0} \pi^{0}$ DECAY}

Using a partial sample of selected $K^{ \pm} \rightarrow \pi^{ \pm} \pi^{0} \pi^{0}$ events $\left(27.33 \times 10^{6}\right)$ the invariant mass of the $\pi^{0} \pi^{0}$ system has been investigated in order to study the formation of pionium atoms. Thanks to the high statistics, the very good $M_{00}^{2}$ resolution and the proper $M_{00}$ reconstruction strategy, the data revealed an anomalous structure in the region $M_{00}=2 m_{+}$, where $m_{+}$is the mass of the charged pion (Fig.3). The cusp structure in the distribution has not been observed in previous experiments. Several checks against instrumental effects have been performed. No evidence for either resolution effects or acceptance non-linearities has been found in the cusp region. In addition, variation in shape of photon energy distribution across the cusp agrees with MC prediction without cusp and no difference is observed between $K^{+}$and $K^{-}$nor between data taken with opposite directions of the magnetic field. The deficit of events in the data in the region $M_{00}<2 m_{+}$is due to a real physical effect.

The observed change of slope suggests the presence of a threshold cusp effect from the decay $K^{ \pm} \rightarrow \pi^{ \pm} \pi^{+} \pi^{-}$contributing to the $K^{ \pm} \rightarrow \pi^{ \pm} \pi^{0} \pi^{0}$ amplitude through the charge exchange process $\pi^{+} \pi^{-} \rightarrow \pi^{0} \pi^{0}$.

The phenomenon has been recently discussed by Cabibbo [9] who computed the $K^{ \pm} \rightarrow \pi^{ \pm} \pi^{0} \pi^{0}$ amplitude taking into account the 1-loop diagram (Fig.4). In the Cabibbo theory, the $K^{ \pm} \rightarrow \pi^{ \pm} \pi^{0} \pi^{0}$ decay amplitude is given by the sum of two terms: the

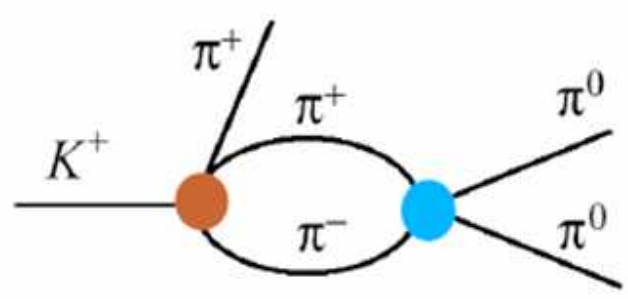

FIGURE 4. $\pi \pi$ 1-loop re-scattering diagram. 
"unperturbed" amplitude $\mathscr{M}_{0}$, á la PDG, plus a new term, $\mathscr{M}_{1}$, proportional to the $I=0$ and $I=2 \mathrm{~S}$-wave $\pi \pi$ scattering lengths (in the limit of exact isospin symmetry). The new term changes from real to imaginary at $M_{00}=2 m_{+}$. The destructive interference of $\mathscr{M}_{0}$ and $\mathscr{M}_{1}$ in the total amplitude causes the cusp and the lack of events below the threshold. More recently Cabibbo and Isidori [10] have computed $\mathscr{O}\left(a_{i}^{2}\right)$ corrections to $K^{ \pm} \rightarrow 3 \pi$ amplitudes including other one-loop and two-loop re-scattering processes diagrams. This model has been used to extract $\left(a_{0}-a_{2}\right)$ from the $\pi^{0} \pi^{0}$ invariant mass distribution with high precision.

Taking into account all systematic and external uncertainties, the final result is:

$$
\begin{aligned}
\left(a_{0}-a_{2}\right) \cdot m_{+} & =0.268 \pm 0.010_{\text {stat }} \pm 0.004_{\text {syst }} \pm 0.013_{\text {ext }} \\
a_{2} \cdot m_{+} & =-0.041 \pm 0.022_{\text {stat }} \pm 0.014_{\text {syst }}
\end{aligned}
$$

The external error is an additional theoretical error of $\sim 5 \%$ on $\left(a_{0}-a_{2}\right)$ due to neglecting higher order terms in the re-scattering model [10]. This uncertainties have no significant effect on $a_{2}$. The two statistical errors from the fit are strongly correlated ( $\rho=-0.858$ ). The result obtained performing the fit with constraints imposed on $a_{0}$ and $a_{2}$ by analyticity and chiral symmetry [11] is:

$$
a_{0} \cdot m_{+}=0.220 \pm 0.006_{\text {stat }} \pm 0.004_{\text {syst }}
$$

which corresponds to

$$
\left(a_{0}-a_{2}\right) \cdot m_{+}=0.264 \pm 0.006_{\text {stat }} \pm 0.004_{\text {syst }} \pm 0.013_{\text {ext }}
$$

This analysis gives the first direct measurement of $a_{2}$, though not as precise as that of $\left(a_{0}-a_{2}\right)$. This result is in very good agreement with Chiral Perturbation Theory $(\chi P T)$ calculations [12], which predict $\left(a_{0}-a_{2}\right) \cdot m_{+}=0.265 \pm 0.004$.

\section{RARE DECAYS: $K^{ \pm} \rightarrow \pi^{ \pm} \pi^{0} \gamma$}

Chiral Perturbation Theory, as a low-energy effective field theory of strong interactions, is a very useful tool to study kaon interactions. The $K^{ \pm} \rightarrow \pi^{ \pm} \pi^{0} \gamma$ decay is important to test high order $\chi P T$ calculations: the dominant part of the amplitude is related to the Inner Bremsstrahlung (IB), however a contribution of the Direct Emission (DE) could be present as well, together with the Interference term (INT) between these two amplitudes.

In the $K^{ \pm} \rightarrow \pi^{ \pm} \pi^{0} \gamma$ decay IB, INT, and DE can be distinguished kinematically using the variable $\mathrm{W}$ which is defined as follows [13]:

$$
W=\frac{\left(P_{K} \cdot P_{\gamma}\right)\left(P_{\pi} \cdot P_{\gamma}\right)}{\left(m_{K} m_{\pi}\right)^{2}}
$$

being $P_{K}, P_{\gamma}, P_{\pi}$ the four-momenta of the kaon, radiative gamma and charged pion, and $m_{K}, m_{\pi}$ the kaon and charged pion masses. The decay rate depends only on $T_{\pi}^{*}$, the energy of the pion in the kaon rest frame, and $W$. Integrating over $T_{\pi}^{*}$ an expression 


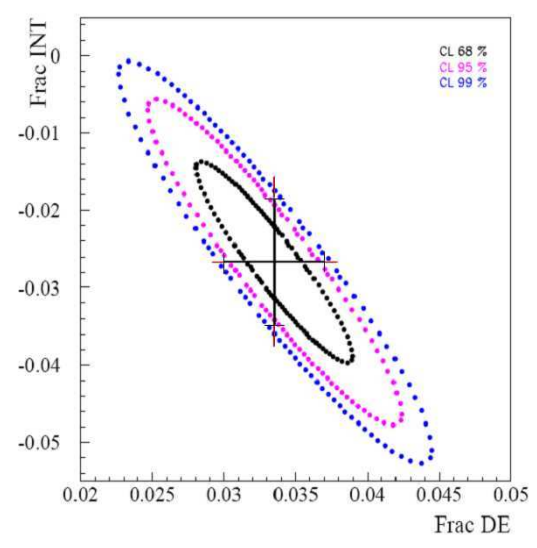

FIGURE 5. Contour plot for DE and INT components.

that separates the different contributions into terms with different powers of $\mathrm{W}$ can be obtained:

$\frac{d \Gamma^{ \pm}}{d W} \approx\left(\frac{d \Gamma^{ \pm}}{d W}\right)_{I B} \cdot\left[1+2\left(\frac{m_{\pi}}{m_{K}}\right)^{2} W^{2}|E| \cos \left(\left(\delta_{1}\right)-\delta_{0} \pm \phi\right)+\left(\frac{m_{\pi}}{m_{K}}\right)^{4} W^{4}\left(|E|^{2}+|M|^{2}\right)\right]$

The three terms represent IB, INT and DE contribution respectively.

An analysis was performed using part of the 2003 statistics: $\sim 2.1 \times 10^{5}$ decays were selected in the regions $T_{\pi}^{*} \leq 80 \mathrm{MeV}$ and $0.2<W<0.9$. At trigger level these events are selected requiring multiple signals in the electromagnetic calorimeter and applying a kinematical cut against $K^{ \pm} \rightarrow \pi^{ \pm} \pi^{0}$ decays. The event reconstruction requires a single charged track $\left(\pi^{ \pm}\right)$, two photons coming from the $\pi^{0}$ decay and an extra photon in time. The global invariant mass has to be compatible with the mass of the charged kaon. Background is reduced to less than $1 \%$ of the DE component applying the following selection criteria: $K^{ \pm} \rightarrow \pi^{ \pm} \pi^{0} \pi^{0}$ decays are suppressed cutting against coalesced gamma events and narrowing the cut around the kaon mass $\left(\mid m_{\pi^{ \pm}} \pi^{0} \gamma-m_{K}<10 \mathrm{MeV} / \mathrm{c}^{2}\right) ; K^{ \pm} \rightarrow \pi^{ \pm} \pi^{0}$ decays are already suppressed at trigger level but to avoid effects occurring at threshold all events have to fulfil the requirement $T_{\pi}^{*} \leq 80 \mathrm{MeV}$.

To obtain the relative contributions of IB, INT and DE a maximum likelihood technique is applied to fit the measured $\mathrm{W}$ distribution to a weighted sum of Monte-Carlo simulated W distributions for IB, INT and DE. The biggest systematic uncertainty is due to the measurement of the trigger efficiencies.

The preliminary results for the measured fractions are (Fig.5):

$$
\begin{aligned}
\operatorname{Frac}(D E) & =\left(3.35 \pm 0.35_{\text {stat }} \pm 0.25_{\text {syst }}\right) \% \\
\operatorname{Frac}(I N T) & =\left(-2.67 \pm 0.81_{\text {stat }} \pm 0.73_{\text {syst }}\right) \%
\end{aligned}
$$

This is the first measurement of a non-vanishing interference term in the $K^{ \pm} \rightarrow \pi^{ \pm} \pi^{0} \gamma$ decay. 


\section{REFERENCES}

1. J. H. Christenson et al., Phys. Rev. Lett. 13, 138 (1964)

2. G. Barr et al. (The NA31 Collaboration), Phys. Lett. B317, 233 (1993)

3. V. Fanti et al. (The NA48 Collaboration), Phys. Lett. B465, 335 (1999)

A. Lai et al. (The NA48Collaboration), Eur. Phys. J. C22, 231 (2001)

J. R. Batley et al. (The NA48 Collaboration), Phys. Lett. B544, 97 (2002)

4. A. Alavi-Harati et al. (The KTeV Collaboration), Phys. Rev. Lett. 83, 22 (1999)

A. Alavi-Harati et al. (The KTeV Collaboration), Phys. Rev. D67, 012005 (2003); Erratum: Phys. Rev. D70, 079904 (2004)

5. S. Eidelman et al. (PDG), Phys. Lett. B592 (2004)

6. A.A. Belkov, A.V. Lanyov, G. Bohm, hep-ph/0311209

E. Gamiz, J. Prades, I. Scimemi, JHEP 10, 042 (2003)

G. D'Ambrosio, G. Isidori, Int. J. Mod. Phys. A13, 1 (1998)

E.P. Shabalin, Phys. Atom. Nucl. 68, 88 (2005)

7. E.P. Shabalin, ITEP preprint 8-98 (1998)

G. D'Ambrosio, G. Isidori, G. Martinelli, Phys. Lett. B480, 164 (2000)

8. W.T. Ford et al., Phys. Rev. Lett. 25, 1370 (1970)

K.M. Smith et al., Nucl. Phys. B91, 45 (1975)

G.A. Akopdzhanov et al. (TNF-IHEP), Eur. Phys. J. C40, 343 (2005)

9. N. Cabibbo, Phys. Rev. Lett. 93, 121801 (2004)

10. N. Cabibbo and G. Isidori, JHEP 503, 21 (2005)

11. G. Colangelo, J. Gasser, H. Leutwyler Phys. Rev. Lett. 86, 5008 (2001)

12. G. Colangelo, J. Gasser and H. Leutwyler Phys. Lett. B488, 261 (2000)

G. Colangelo, J. Gasser and H. Leutwyler Nucl. Phys. B603, 125 (2001)

13. J. D. Good, Phys. Rev. 113, 352 (1959) 